\title{
Design and manufacture of PM2.5 measuring instrument Based on STC12C560S2
}

\author{
Hui Zhang ${ }^{1, \text { a }}$, Yani Fan ${ }^{1, b}$ and Jian-hao Yuan ${ }^{1, c}$ \\ ${ }^{1}$ Department of Physics and Information Engineering, Guangdong University of Education, Guangzhou \\ 510303, China; \\ azhanghui27@126.com, bfanyani@gdei.edu.cn, cyuanjianhao@163.com
}

Keywords: STC12C560S2-MCU, SHARP-GP2Y1010AU0F, Optical dust sensor.

\begin{abstract}
A fundamental problem in air pollution is to find an easy method to check air pollution index. This article describes how to design a portable or handheld air quality detector based on STC12C560S2 microcontroller. The SHARP-GP2Y1010AU0F optical dust sensor is used to detect the concentration of air dust, and air quality index(AQI) can be acquired on the LCD monitor. The development of hardware system is realized based on the selection of modules such as MCU, sensor, dot-matrix liquid crystal display, lighting alarm, serial communication and mobile power. The functions such as data detection and processing, key control, result display, overrun alarm, data storage and historical data query are completed.
\end{abstract}

\section{Introduction}

In recent years, with the development of urbanization, air quality problem is getting worse and worse. With the increase of PM2.5 concentration, the damage to the body becomes more and more serious. PM 2.5 means fine aerosol particulate matter with an aerodynamic equivalent diameter less than 2.5 um in air. The longer they are suspended in air, the more serious air pollution is. PM2.5 has the characteristics of small particle size, large area, strong activity, easy to adsorb heavy metals and other toxic substances. In prior art, the detection methods includes a weight detection method, a micro oscillation balance method, a radiation detection method and a light scattering detection method.

Although the air quality monitoring points in China have expanded from city to countryside, moreover, we can check the air quality information from Internet, but the air quality information is an average value from a wide range of monitoring points, a small area of air quality information still cannot be achieved.

In order to quantitatively describe the air quality, the paper introduces the air quality index. Air quality index (AQI) is dimensionless, can quantify the air quality status ${ }^{[1]}$.

The air quality index (AQI) of pollutants is calculated as follows ${ }^{[2-3]}$ :

$$
I_{x}=\frac{I_{H i}-I_{L o}}{C_{H i}-C_{L o}}\left(C_{x}-C_{L o}\right)+I_{L o}
$$

Where: $I_{x}$ : the air quality sub-index;

$$
\begin{aligned}
& C_{x} \text { : Concentration of harmful gases; } \\
& C_{H i} \text { : Close to the upper limit of pollutant Cx concentration, a constant; } \\
& C_{L o} \text { : Close to the lower limit of Cx pollutant concentration, a constant; } \\
& I_{H i} \text { : The air quality sub-index corresponding to Chi, a constants; } \\
& I_{L o} \text { : The air quality sub-index corresponding to Clo, a constants; }
\end{aligned}
$$

The formula (1) can be transformed into formula(2) as follows: 


$$
\frac{I_{\chi}-I_{L o}}{I_{H i}-I_{L o}}=\frac{C_{X}-C_{L o}}{C_{H i}-C_{L o}}
$$

The air quality index is calculated as follows:

$$
A Q I=\max \left\{I_{1}, I_{2}, I_{3} \ldots I_{n}\right\}
$$

Where: $I$ : the air quality sub-index.

\section{Organization of design}

\subsection{Overall plan}

The design of a handheld air quality detector is shown in Fig.1. Air is measured by the sensor modules named SHARP-GP2Y1010AU0F, which sends detected voltage values to MCU STC12C5A60S2. Data analysis and processing are implemented by MCU, and the corresponding information of the ambient air quality assessment and health assessment is sent to LCD. If the detected value exceeds the pre-set alarm value, the light alarm module will respond. Users can control the MCU reset, data storage and communication. Power supply module provides adequate power.

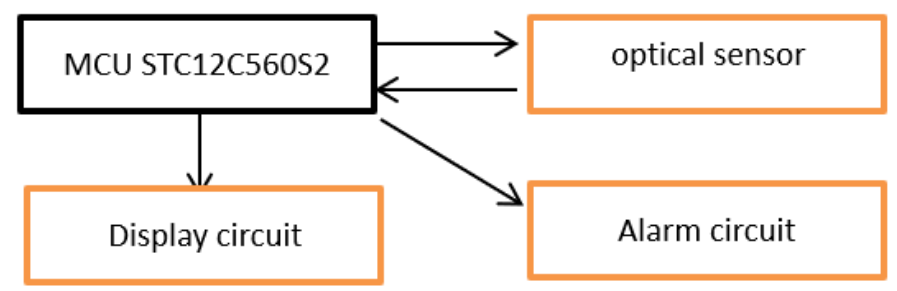

Fig.1 the overall design diagram

An optical detection sensor produced by Sharp is adopted in this design. This sensor uses the principle of photosensitive principle which detects optical signal reflected by dusts in the air and converts to electrical signals. According to the particle concentration, the sensor outputs pulse height to a certain linear relationship and determines the concentration of particulate matter content. Sensor technical parameters ${ }^{[4-5]}$ :

Operating Voltage: 0.3-7.0 V

Operating Temperature: $-10^{\circ} \mathrm{C}$ to $65^{\circ} \mathrm{C}$

Operating Current: 20mA

Effective Receptive Angle: $\pm 60^{\circ}$

\subsection{Operation Principle}

In this design, the main role of MCU is to receive the analog signals of sensor and convert them to digital. According to the requirements of LCD drive, MCU produces a pulse signal by period $\mathrm{T}=10 \mathrm{~ms}$, bandwidth $\mathrm{BW}=0.32 \mathrm{~ms}$, sampling time $\mathrm{t}=0.28 \mathrm{~ms}$, at the same time sends a digital signal to LCD 1602.

The flow chart is shown in Fig.2, MCU sends a start command to the dust sensor, and samples at the same time. The dust concentration limit settings are set by software. The real-time PM2.5 values are displayed and an alarm is given if necessary.

A small fan and a bracket are designed on the basis of original sensor. MCU is mainly responsible for data sampling and data output. A mobile power provides sufficient power.The circuit diagram is shown as Fig.3. 


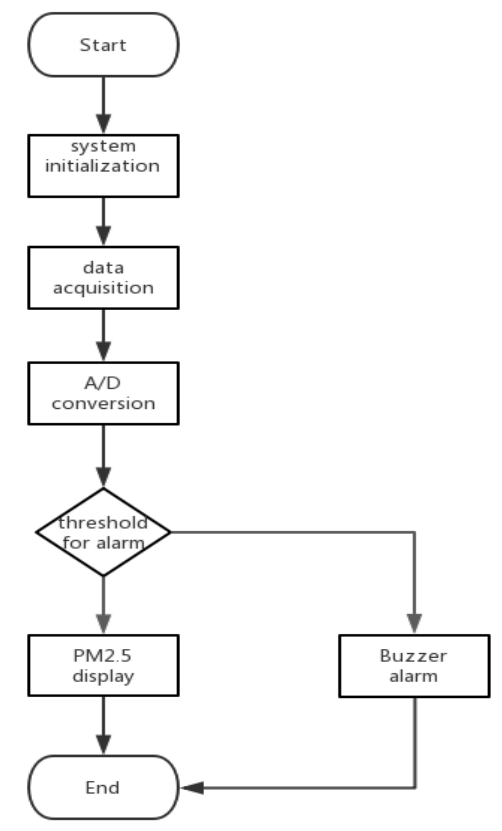

Fig.2 the Flow Chart

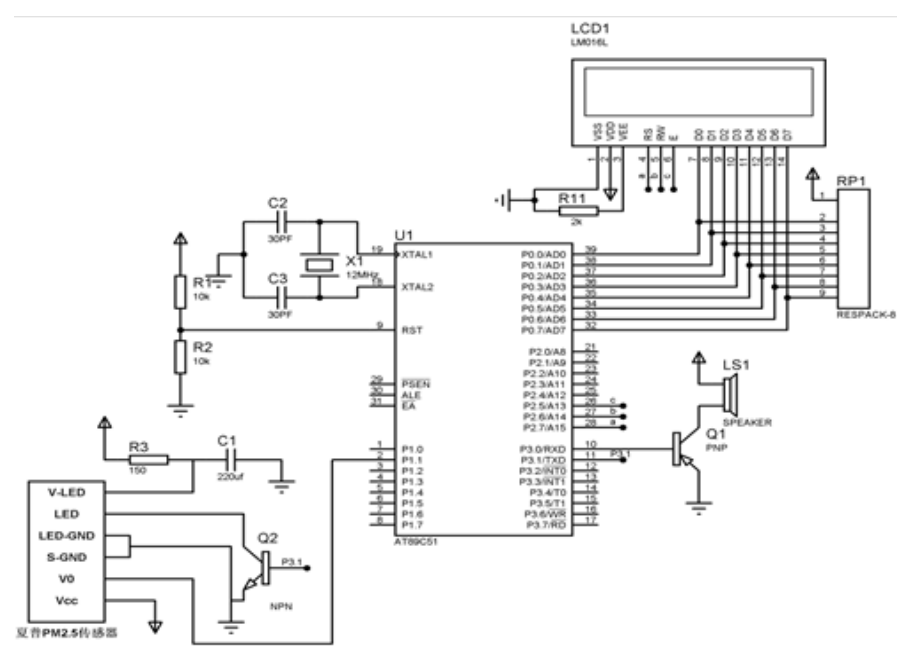

Fig.3 the Circuit Diagram

\section{Debugging}

\subsection{Test Plan}

According to the instructions of dust sensor, the sensor will be stable within 1 second after power on. The output voltage represents the dust concentration. We can also discriminate the kinds of objects based on the time of output levels, and distinguish the kinds of dusts by the change of dust concentration in a certain period of time.

\subsection{Test Equipment}

Test equipment include voltage values of each port, conduction of the components, sensor pulse signals and output signals. Therefore, Multimeter and DS1062E-EDU oscilloscope are needed.

\subsection{Testing Results}

The measured pulse waveform of the control LED is shown in Fig.4. The measured waveform of A / D sampling is shown in Fig.5. The sampling waveform in the manual is shown in Fig.6. Compared Fig.5 with Fig.6, it is easy to conclude that the measured waveforms from designed testing instrument and those from manual are basically consistent. The experimental results are reasonable.

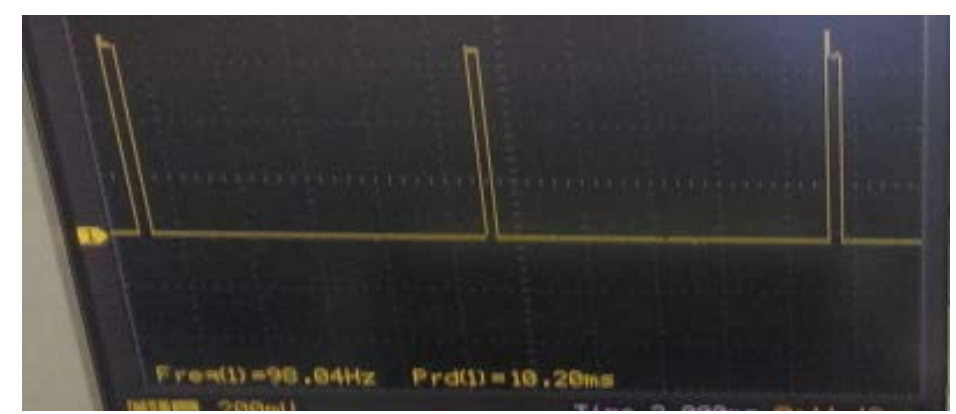

Fig.4 LED pulse signal waveform 


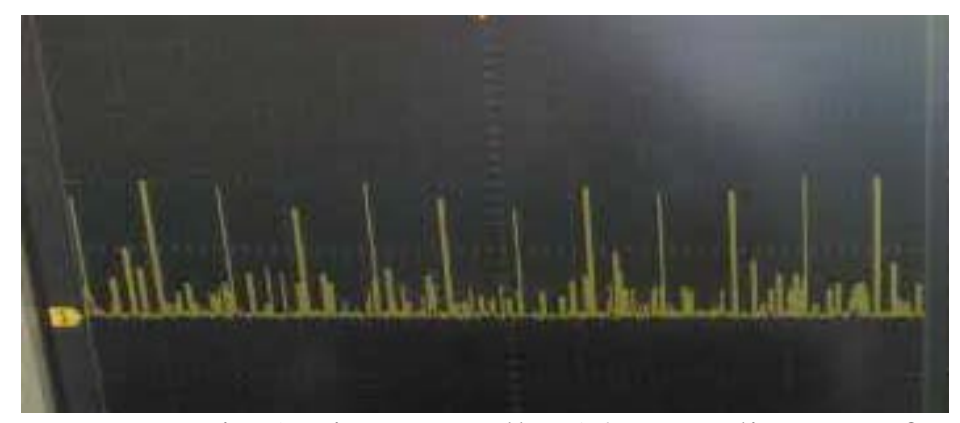

Fig.5 Microcontroller A/D sampling waveform

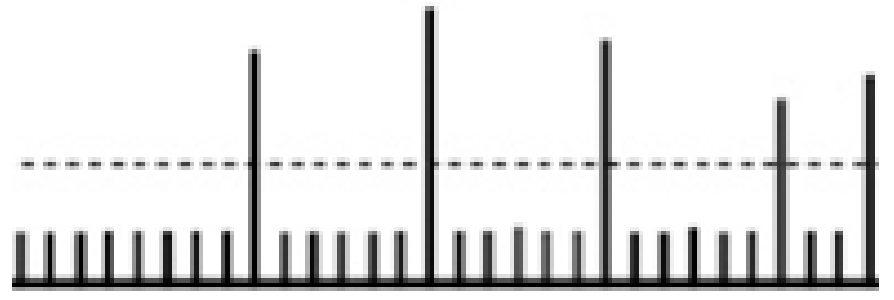

Fig.6 Theoretical sampling waveform

\section{Summary}

The system, compared with the traditional STC-51 microcontroller, STC-12 microcontroller can quickly capture data and calculate results during the very short high-level output time, what's more, no external A / D converter is needed. The entire cost of the instrument is about 35 RMB. Based on the features of STC-12 microcontroller, other external functions can be also expanded, such as displaying real-time indexes of $\mathrm{PM} 10, \mathrm{O}_{3}$, CO etc. and providing health devices to uses.

In the market positioning, this design tends to be used as a teaching instrument because of its low cost. It can be used as a basic experiment tool in electronic engineering courses. The contents of these courses can be related to programming, welding and layout design. In addition, based on the advantages of STC-12 microcontroller, extensional design can be carried out so as to stimulate students' interest and guide students to learn more effectively from practice.

\section{Acknowledgments}

Project supported by the Characteristic innovation projects of Guangdong ordinary university in 2016, China (No. 2016GXJK126).

\section{References}

[1] Impact of urbanization level on urban air quality A case of fine particles (PM2.5)in Chinese cities. Han L J,Zhou W Q,Li W F,et al. Environmental Pollution . 2014

[2] Estimation of Atmospheric Dust Deposition on Plant Leaves Based on Spectral Features[J] . X. Yan,W. Shi,W. Zhao,N. Luo. Spectroscopy Letters . 2014 (7)

[3] Atmospheric heavy metals and Arsenic in China: Situation, sources and control policies[J] . Jingchun Duan,Jihua Tan. Atmospheric Environment . 2013

[4] Large scale air pollution estimation method combining land use regression and chemical transport modeling in a geostatistical framework. Akita Y,Baldasano J M,Beelen,R et al. Environmental Sciences . 2014 